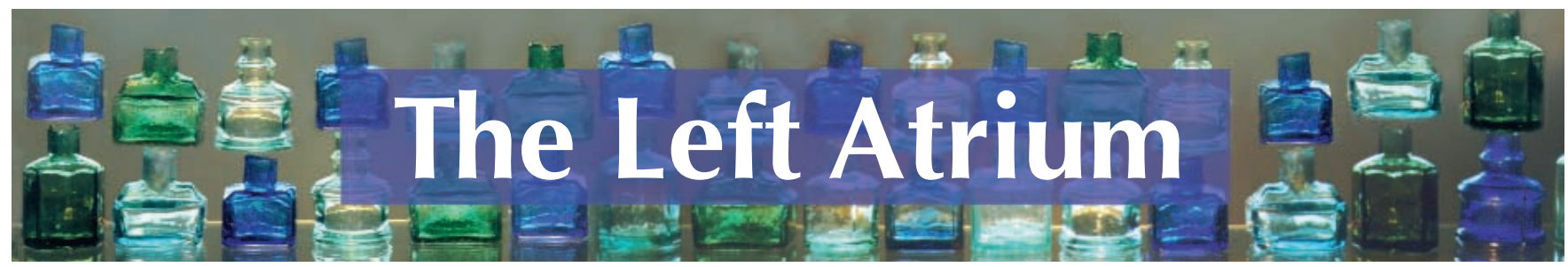

\section{Digital bodies}

\section{Corpus callosum}

Written, directed and produced by Michael Snow

Directors of photography: Robbi Hinds and Harald Bachman

Editor: Paul Cormack

Special-effects supervisor: Greg Hermanovic

Senior animator: Rob del Ciancio

Canada, 2002; filmed on digital video; 92 minutes

$\mathrm{T}$ The films of Michael Snow — artist, filmmaker, musician and arguably the most prolific Canadian artist on the international scene (he received the Order of Canada for his work, and his geese sculptures adorn the Eaton Centre in Toronto) - have always been reserved for the art crowd; it takes effort to understand them and considerable willpower to watch them, for they tend to be long, repetitive and rather soporific. In 1971 Snow went to northern Quebec and set up a stationary automated roving-eye camera that scanned the landscape for 24 hours. He edited the footage down to four hours, for the ultimate self-referential "in-the-eye-ofthe-beholder" statement on visual perception (La region centrale). In 1967 he spent 45 minutes focusing on a wave in a picture on the wall of a nondescript New York City loft (Wavelength). His films deflect the conditions necessary for the "suspension of disbelief" by making the viewer acutely aware of how he or she has been conditioned to construct meaning from the medium of film. Corpus Callosum mounts a frontal attack on our culture's obsessive love affair with motion pictures by experimenting with their current digitized incarnation.

The corpus callosum is a band of white fibres (axons) that interconnect cortical neurons from the right and left cerebral hemispheres. Severing the white fibres between the two hemispheres, which is sometimes done to treat intractable epilepsy, leads to interesting clinical syndromes in which each hemisphere seems to function indepen- dently of the other. Both hemispheres need a certain amount of cross-talk to make sense of the totality of experience. Snow chose Corpus Callosum as his title to reflect the film's intermediary status between fact and fiction, the artificial and the natural. Although critics have both praised the film as groundbreaking and dismissed it as a colossal 92-minute waste of time, the affinities between the structure of the nervous system and the seamless, warm digital cocoon that envelops us at the movies are the targets of Snow's cinematic antics.

The film begins with the actors' entrance through a door marked "Corpus Callosum." Snow then proceeds to systematically construct, deconstruct and reconstruct each frame by showing us the digital morphing of time, space and narrative. Although there is a sound track consisting of the hum of white noise, the director's voice is clearly audible in the background. The setting is partitioned into a bland office, where each worker sits at a computer screen, and a domestic living room, where three actors enact a nuclear family. The woman in the domestic scene morphs from office worker to wife to cross-dresser to pregnant woman to transsexual. Although Snow's digital attacks on her demonstrate that what we are seeing is a hoax, the viewer still hopes that somehow she will guide us out of this jungle of digital trickery. We end up identifying with the actors, not as characters in a story, but simply in hoping that the director will stop these childish tricks.

The camera pans continuously from left to right in a slow movement that is re-looped so that the effective space is compressed and flattened: again, this is an anti-narrative device. As viewers we are painfully aware of the cameraman rolling the film in this futile manner.

The action is minimal: the actors stare at their computers or engage in trivial interactions with each other. Yet extraordinary things happen to them — digitally — as the director commands them to do impossible things. At one point he yells "Corner!" at which point the group is digitally squashed into one corner of the frame; with "Release!" they all fall to the ground. Moustaches are painted on their faces as they converse with one another; they are told to reach for objects that then disappear. At times there seems to be some complicity between audience and director, but in the end the viewer, like the actors, becomes a victim of these gags by repeatedly being jolted back into reality.

Snow's manipulation of cinematic variables creates a longing for our perceptual apparatus to retreat to the familiar and relieve the dysphoria provoked by these uncomfortable digital effects. At one point, Snow lets us see the actual film layer as he twists and then inverts it. This warping of the film itself does not dampen our hope for perceptual or psychological salvation. The movie ends with the listing 
of credits and the closure (and relief!) expected with the restoration of the "real" reality - only to restart again, reopening the story that wasn't a story to begin with. The action resumes even more childishly, with endless domestic scenes in which the director instructs the actors to reach out and grasp a cup, only to frustrate the (real or digitized) actor by making the cup disappear.

What is Snow saying about the status of flesh-and-blood actors in a digitized movie industry? Movies have always been "fake," (think of early car chase scenes shot against a moving background), but this did not prevent us from identifying with the actors in the story. Without the sense-defying digital effects of today's epic films (Lord of the Rings, The Matrix) the main characters appear impotent. In Corpus Callosum there is no story, no characters to identify with; all special effects are laid bare, and we feel compelled to fill in the blanks. As the special-effects software programmer shares equal credit with the director, writer and actors, will the narrative power of film be enhanced, or reduced?

Snow ends the film (for real, this time) with a scene in which he joins a few others in a private auditorium to view his
1956 film, made at the start of his career, of an animated drawing of a stick figure. This is where it started for him - and, in Corpus Callosum, where he is right now. As the meaning of "real" is morphed by the digital revolution and we retreat into deeper layers of cyberspace, will the movies, as a mirror of society, raise consciousness or anesthetize us like a severed corpus callosum? His message might be quite simple: enjoy the show, but don't believe everything you see.

\section{Normand Carrey}

Psychiatrist

Halifax, NS

\section{Room for a view}

\section{Napalm}

Thave never smelled napalm, and I 1 pray I never will. But I have never arrived on the wards in the morning without being reminded of it, or rather of Robert Duvall's famous quip from Apocalypse Now. But the smell of the wards in the morning is the smell of life itself, the smell of human excrement.

And so perhaps on the morning in question the pungent odour was the only familiar to which I could cling; certainly, I remember it vividly. I was

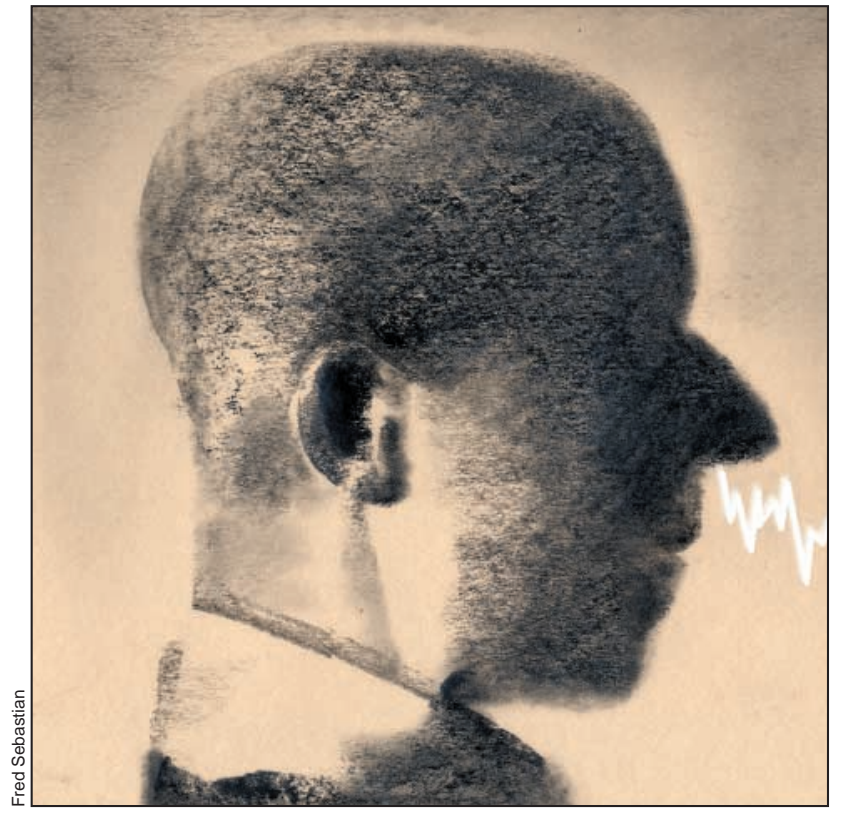

freshly turned out in my crisp white clinical jacket (not coat), timidly and confusedly attempting to navigate through one of my city's crumbling teaching hospitals in search of a patient whose name has long since escaped me.

It was the start of second-year medicine. At home I enjoyed parading in front of mirrors in my little white jacket, but now I was nervous and perspiring, conspicuous and clumsy in my cloak of no colour but many implications (to those who know). Pockets bulging, clipboard in hand and bag of diagnostic paraphernalia slung awkwardly over my shoulder, I entertained the vain hope that perhaps the patient might already have been discharged. But that would have been too easy, and easy isn't always best.

My assignment: CPA, comprehensive patient assessment, wherein neophyte not-yet-clerks interview, examine and "present" patients to a group of classmates and an attending, the patients having been semi-coerced into volunteering their stories and bodies in the service of medical education. It turned out my patient was on the ortho ward, a seventy-something gentleman, post-op day 1 following RTKA (whatever that was) according to the virtually illegible scribbles in the cryptic, cluttered, and (secretly) colour-coded document known as "the chart." I fumbled my way through the dilapidated binder and discovered that TKA (obviously) referred to total knee arthroplasty (and R to the opposite of L; very impor$\operatorname{tant).~Okay,~the~knee~in~317.~Off~I~went~}$ to discharge my duty if not my patient.

And now the smell to which I earlier referred, powerful and fresh, reminded me that the gentleman whose acquaintance I was about to make was perhaps as exposed and vulnerable as I was in this encounter. "Mr. Knee?" I asked, trying to sound confident. In response to his affirmative reply I introduced myself too quickly and sought to remind him that he had agreed to be interviewed by a medical student, and that I, the lines on my face and grey in my hair notwithstanding, was that student.

"Sure, sure, come on in," groaned and grimaced Mr. Knee. ${ }^{*}\left({ }^{*}\right.$ Not his real name.) "I'm having some awful heart- 